\title{
Muerte prematura y discapacidad en los derechohabientes del Instituto Mexicano del Seguro Social
}

\author{
Gabriela Rodríguez-Abrego, MC, EsE,(1) Jorge Escobedo de la Peña, MC, MSP, MCE, (2) \\ Beatriz Zurita, MC, MAE, PhD, ${ }^{(3)}$ Teresita de Jesús Ramírez, LEO, MCSS. ${ }^{(1)}$
}

\author{
Rodríguez-Abrego G, Escobedo de la Peña J, \\ Zurita B, Ramírez TJ. \\ Muerte prematura y discapacidad en los derechohabientes \\ del Instituto Mexicano del Seguro Social. \\ Salud Publica Mex 2007;49:132-143.
}

\section{Resumen}

Objetivo. Realizar estimaciones de la carga de la enfermedad para 129 causas a fin de identificar prioridades de salud en cada una de las delegaciones y presentar datos comparativos entre 1995-2000. Material y métodos. Se analizaron los indicadores años de vida saludables perdidos (AVISA) y esperanza de vida saludable (EVISA) de los años 1995 y 2000 en la población derechohabiente del Instituto Mexicano del Seguro Social (IMSS); en ambos estudios se utilizó la metodología del Estudio Mundial de Carga de Enfermedad. Los datos de 1995 se analizaron en 1997, y los datos de 2000 , en 200I. El estudio comparativo se llevó a cabo en 2006 en el IMSS, México. Resultados. La mayor proporción de AVISA perdidos se debió a enfermedades crónicas, si bien la tasa en 2000 es I.5 veces mayor respecto de 1995. Las prioridades identificadas fueron diabetes mellitus, cardiopatía isquémica y la enfermedad cerebrovascular con pérdidas principalmente por muerte prematura, así como padecimientos asociados con discapacidad con una importante carga de enfermedad, tales como el SIDA o la depresión.Aún se observan rezagos epidemiológicos. Conclusión. La población derechohabiente se encuentra aún en fase de transición epidemiológica, lo que favorece una polarización en las condiciones de salud. Es necesario que las políticas de salud se orienten a dar una mejor respuesta y a revertir las tendencias de padecimientos que representan un riesgo institucional para el financiamiento de la atención de los derechohabientes.

Palabras clave: años de vida ajustados por discapacidad; esperanza de vida saludable; México
Rodríguez-Abrego G, Escobedo de la Peña J,

Zurita B, Ramírez TJ.

Premature death and disability profile in the population affiliated to the Mexican Institute of Social Security (IMSS). Salud Publica Mex 2007;49:132-143.

\section{Abstract}

Objective:To carry out estimations of the burden of disease for 129 causes in order to identify health priorities in the different geographic regions of the country and to present comparative data between 1995 and 2000. Material and Methods. Indicators such as disability-adjusted life years (DALYs) and disability adjusted life expectancy (DALE) were analyzed for the population covered by IMSS in 1995 and 2000; for both years, the methodology proposed by the Burden of Disease Worldwide Study was applied. Data corresponding to 1995 were analyzed in 1997 , while data corresponding to 2000 were analyzed in 2001 . The comparative study was carried out in 2006 by IMSS, Mexico. Results. The higher proportion of DALYs was due to chronic diseases, although the 2000 rate is I.5 times higher than that of 1995. Priorities that were identified were diabetes mellitus, ischemic cardiopathy, and cerebrovascular disease, with a loss that is mainly accounted for by premature death, as well as by diseases related to disability with an important burden of disease, such as AIDS or depression. Epidemiological backlogs can still be observed. Conclusion. The population covered by IMSS is still in a phase of epidemiologic transition, favoring polarization in health conditions. Health policies need to be directed toward bringing about an improved response and reversing the trend in diseases that represent an institutional risk for the financing of health care.

Key words: disability-adjusted life years; disability-adjusted life expectancy; México

(I) Coordinación de Planeación y Desarrollo, Instituto Mexicano del Seguro Social (IMSS). México DF.

(2) Unidad de Investigación en Epidemiología. Clínica Hospital General Regional No. I Gabriel Mancera, IMSS. México DF.

(3) Coordinadora de Planeación y Desarrollo, Dirección de Prestaciones Médicas, IMSS.

Fecha de recibido: 27 de septiembre de 2006 - Fecha de aprobado: 22 de enero de 2007 Solicitud de sobretiros: Mtra. Gabriela Rodríguez. Hospital General Regional Gabriel Mancera, IMSS, Unidad de Investigación Epidemiológica. Gabriel Mancera esquina Xola, col. del Valle. México, DF, México.

Correo electrónico: gabriela.rodrigueza@imss.gob.mx 
M éxico fue uno de los primeros países en desarrollo en estimar la carga nacional de la enfermedad mediante el uso de los años de vida saludable perdidos ajustados por discapacidad (AVISA). Los resultados de esta estimación se han empleado en la planeación de los servicios de salud; ${ }^{1}$ de manera particular, cabe destacar las contribuciones del Estudio Economía y Salud realizado en México en 1994 que dio pauta a la elaboración de políticas de salud para grupos específicos. $^{2}$ Entre 1990 y 2003 se instrumentaron diversos programas, tales como el Programa de Apoyo a los Servicios de Salud para Población Abierta (PASSPA), el Programa de Ampliación de Cobertura (PAC) y el Programa de Extensión de Cobertura (PEC), con el objetivo de extender la cobertura a grupos socialmente desprotegidos mediante la provisión de aproximadamente 13 paquetes básicos de intervenciones de salud. De manera más reciente se ha instrumentado el Programa del Seguro Popular, que consiste en un modelo de aseguramiento mediante financiamiento público dirigido a la población de bajos recursos económicos. El Seguro Popular incorpora 91 intervenciones seleccionadas por su costo-efectividad. ${ }^{3}$

El concepto de AVISA ha sido usado con frecuencia desde la publicación del Informe del Desarrollo Mundial 1993 del Banco Mundial, ${ }^{4}$ en el que se habla ya de manera recurrente de la "carga global de la enfermedad". Este término adquiere una naturaleza todavía más conspicua en el informe de 1996 del Comité Ad Hoc para la Investigación en Salud de la Organización Mundial de la Salud y en el Estudio Mundial de Carga de Enfermedad. 5,6 Se trata de una medida de resumen que conjunta las pérdidas por morbilidad o mortalidad por causas específicas e incluye la contribución que genera la gravedad de las consecuencias funcionales de la enfermedad. La estimación de la carga de la enfermedad mediante los AVISA permite establecer prioridades en la atención de la salud no identificables con el solo uso de indicadores individuales tales como la morbilidad o la mortalidad. ${ }^{7}$ Asimismo, contribuye a identificar el perfil de salud de la población y los cambios en las principales causas de enfermedad y muerte que ocurren entre poblaciones en el curso del tiempo. Este proceso, conocido como transición epidemiológica, comprende tres aspectos fundamentales: a) el cambio en el patrón de enfermedades asociadas con procesos infecciosos y deficiencias nutricionales por enfermedades crónicas, lesiones y padecimientos psiquiátricos; b) el desplazamiento de la mortalidad en niños hacia los adultos; c) el cambio en el significado social de la enfermedad (evento agudo que se resuelve mediante curación o concluye con la muerte para convertirse en un proceso crónico asocia- do con fenómenos sociales, psicológicos y económicos crecientes).

Otro indicador compuesto que se utiliza en la medición de la carga de la enfermedad es la esperanza de vida ajustada por discapacidad (EVISA), que mide el número equivalente de años de vida que se espera sean vividos con salud completa, es decir, libre de enfermedad, o la esperanza de vida saludable. Su uso ha permitido establecer comparaciones y medir rezagos en materia de salud. ${ }^{8,9}$

Para identificar el efecto que ha tenido el cambio en el patrón de comportamiento de las enfermedades en la población derechohabiente del IMSS es necesaria la medición de la muerte prematura y la discapacidad en la misma, es decir, toda alteración de la salud que represente una pérdida de bienestar. Estas alteraciones pueden derivar de enfermedades, muerte, factores de riesgo y secuelas asociadas con discapacidad.

La primera estimación de la carga de la enfermedad en la población cubierta por el IMSS se llevó a cabo en 1997 con información de 1995, y este ejercicio se repitió cinco años más tarde con información del año 2000.

El objetivo de esta publicación es presentar los resultados comparativos de ambas estimaciones para 129 causas, incluyendo la comparación del establecimiento de prioridades a partir del uso de información sobre morbilidad, mortalidad y los AVISA en los niveles nacional y delegacional. Asimismo, busca presentar las diferencias entre las delegaciones del Instituto en el nivel nacional* mediante la comparación de la contribución de los años perdidos por muerte prematura (APMP) y aquéllos por discapacidad para la construcción de los AVISA. Por último, mediante la comparación de la esperanza de vida y la esperanza de vida saludable (o su contraparte, la esperanza de vida con discapacidad) en las delegaciones, se establecen patrones de polarización epidemiológica dentro de la transición en salud que experimenta el país.

\section{Material y métodos}

Con base en la información de las estadísticas nacionales del Instituto Mexicano del Seguro Social, se llevó a cabo un análisis comparativo entre los años 1995 y 2000 de las defunciones correspondientes a los años de estudio, la estructura poblacional de los derecho-

\footnotetext{
* Las delegaciones responden al sistema de regiones territoriales que establece el propio IMSS según su organización administrativa. En muchos casos, coinciden con las entidades federativas en las que está dividido oficialmente el país.
} 
habientes por grupos de edad y sexo, las causas de consulta y los egresos hospitalarios. La información correspondiente a estos dos años se unificó con criterios metodológicos a fin de que los datos recabados resultaran comparables.*

Como es sabido, la estimación de la carga de la enfermedad requiere que la información satisfaga algunos criterios de validación, calidad de la información y uniformidad. De acuerdo con ello se llevó a cabo la reclasificación de las causas de muerte según los criterios de la Clasificación Internacional de Enfermedades, novena y décima revisiones, ${ }^{10,11}$ que consideran los padecimientos incluidos en el estudio de la carga global de enfermedad. ${ }^{12,13}$ Estos padecimiento se clasificaron en tres grupos de enfermedad: Grupo I, referente a las enfermedades transmisibles y las afecciones maternas y perinatales; Grupo II, a las enfermedades no transmisibles; y Grupo III, a las lesiones.

Asimismo se procedió a realizar la corrección del subregistro y la mala clasificación de causas de muerte en 129 causas (112 causas de enfermedad y 17 residuales). Las causas no específicas fueron reubicadas según la naturaleza del diagnóstico, lo que redujo el porcentaje de datos residuales según lo indican los procedimientos para la validación de causas y métodos de ajuste. ${ }^{14}$

A fin de calcular la mortalidad infantil (probabilidad de morir antes de un año expresado por mil nacidos vivos) se utilizó el método indirecto; para ello se obtuvieron inicialmente las tasas de fecundidad específicas, el promedio de hijos nacidos vivos, de fallecidos y de sobrevivientes según edad de la madre para cada entidad federativa. La edad media de la maternidad se calculó de acuerdo con información de la Encuesta Nacional de Dinámica Demográfica (1993), con datos extrapolados a 1995, y los datos del Censo Nacional de Población y Vivienda para el año 2000. La proporción de madres por grupos quinquenales de edad sirvió para estimar el promedio de niños que han muerto, como una medida de la mortalidad, desde el nacimiento hasta cierta edad. Este método, según el procedimiento original de Brass, ${ }^{15}$ concierne a modelos de mortalidad y fecundidad utilizados para calcular los factores que trasforman la proporción de hijos fallecidos en probabilidades de morir. Posteriormente se calcularon las muertes de niños de 1 a 4 años.

\footnotetext{
* El estudio de 1995 fue conducido por la Fundación Mexicana para la Salud en 1997, mientras que el estudio de 2000 fue realizado por la Universidad Javeriana en 2001 con financiamiento del Banco Mundial.
}

La propuesta original del estudio de Carga Mundial de Enfermedad utilizó el método de tablas vida de Coale \& Demeny Oeste $26,{ }^{16}$ lo que sugirió la conveniencia de adoptar la misma metodología y conseguir con ello la comparabilidad de datos con otros estudios. El modelo de Palloni-Heligman presenta, respecto de otras modificaciones del método de Brass, la ventaja de que utiliza patrones de fecundidad del modelo propuesto por Coale y Trusell, ${ }^{17}$ los cuales se caracterizan por ajustarse a una amplia gama de experiencias observadas de fecundidad, además de que utiliza patrones de mortalidad del modelo de tablas de vida de las Naciones Unidas, basadas en datos de países en desarrollo. No obstante estas ventajas, las diferencias entre los resultados obtenidos con uno y otro método no son significativas, por lo que se optó por la ventaja de la comparabilidad.

La estimación de la mortalidad por entidad federativa se llevó a cabo una vez seleccionado el patrón de mortalidad. Para ello se utilizó el componente CEBS (Children ever born and children surviving) del programa MORTPAK lite. ${ }^{18}$ Las tasas ajustadas de mortalidad se obtuvieron con los datos calculados previamente. En la construcción de las Tablas de Vida se utilizaron las tasas específicas de mortalidad $\mathrm{nMx}$ por grupos quinquenales; se calculó nqx la probabilidad de morir y el resto de las funciones de la tabla hasta llegar a la esperanza de vida de los diferentes grupos de edades ex. ${ }^{19}$

A fin de evaluar el efecto de la transición epidemiológica se utilizó la metodología propuesta por la Fundación Mexicana para la Salud (Funsalud), que hace uso del indicador de probabilidad de morir, indicador que presenta una sensibilidad simultánea al rezago epidemiológico (mortalidad en la infancia) y a los cambios emergentes (mortalidad en adultos). Dicho indicador permite estratificar la mortalidad en grupos y se basa en la relación entre la probabilidad de morir de los menores de cinco años (5q0) y la probabilidad de morir de los adultos de 15 a 45 años (45q15). En este caso, se estableció la comparación entre las condiciones que presentaban dicha relación en 1995 y las correspondientes al año 2000. ${ }^{20}$

Dado que los datos requerían de un manejo estadístico que permitiera sustentar la ubicación de los estratos según el grado de transición de la mortalidad en las diferentes entidades federativas, se tomó como parámetro de comparación el promedio nacional de las tasas de mortalidad infantil y en adultos. Para ello se identificaron los diferentes estratos por arriba y por debajo de este valor. En el caso de los estados con mortalidad infantil menor al promedio nacional se identificaron dos grupos: aquellos ubicados en el primer 
cuartil, que son los que presentan las tasas de mortalidad más bajas y se ubican, por tanto, en fase de transición avanzada, y aquellos que presentan tasas de mortalidad por arriba del primer cuartil y por debajo del promedio nacional, que son los que se ubican en fase de transición intermedia.

Por lo tanto, las delegaciones del IMSS se dividieron de acuerdo con la probabilidad de morir de los menores de cinco años y la probabilidad de morir de los adultos, ambos dentro del promedio nacional y en el cuartil 25. A partir de esto se obtuvieron seis regiones en el país: a) Región de Transición Avanzada, que presenta una mortalidad en la infancia por abajo del cuartil 25; b) Región de Transición Intermedia, con una mortalidad infantil ubicada entre el cuartil 25 y la media; c) Región de Transición Media o Incipiente, con una mortalidad en la infancia por abajo del promedio nacional y una mortalidad en adultos por arriba del promedio; d) Región de Rezago Infantil, que se caracteriza por registrar una mortalidad en la infancia por arriba del promedio nacional; e) Región de Rezago moderado, que presenta una mortalidad infantil por abajo del promedio nacional y una mortalidad en adultos por arriba del promedio; y f) Región con Rezago Extremo, con una mortalidad infantil y de adultos por arriba del promedio nacional.

Para la estimación de APMP se utilizaron las defunciones corregidas por causa, edad y sexo. Los datos se estandarizaron con la tabla de vida de Coale Demeny modelo Oeste nivel 26, que representa una sociedad de baja mortalidad con una esperanza de vida al nacer de 82.5 años para las mujeres y de 80 años para los hombres; con ello se eliminan las particularidades de la estructura de población que presenta esas esperanzas de vida. Dado que la esperanza de vida en hombres y mujeres es diferente en todas las sociedades del mundo, se toma un límite de vida diferente según el género.

Para el cálculo de los años vividos con discapacidad (AVD) se utilizó el programa de cómputo DISMOD II, que sirvió para corregir los casos incidentes. Con tal propósito se construyó un modelo epidemiológico que dio consistencia interna a la información epidemiológica disponible de incidencia-prevalencia-mortalidad. Los casos incidentes corregidos para cada padecimiento en el periodo de referencia, la edad de inicio y el tiempo de duración de cada enfermedad se multiplicaron en una hoja de cálculo utilizando la metodología de cuantificación de la discapacidad. ${ }^{12}$ Los AVISA se obtuvieron de la suma de los años perdidos por muerte prematura y los años vividos con discapacidad. A estos cálculos se aplicó una tasa de descuento de $3 \%$ en virtud de la necesidad de actualizar los años de vida saludables que se perderían en el futuro con valores presentes. La aplicación de tasas de descuento reconoce que el objeto de medición del indicador es el tiempo perdido en el futuro debido a una enfermedad o una lesión que, aun cuando ocurra ahora, puede prolongarse por tiempo indefinido, ya sea en forma de secuela, recaída o complicación. Al respecto, si se acepta que la diferencia entre el presente y el futuro es pequena, es conveniente que dicha tasa no sea superior a $3 \%$.

Una vez calculadas las tablas de vida para la población derechohabiente del IMSS se procedió a calcular la EVISA a nivel nacional y por delegaciones para los años 1995 y 2000. Para ello se utilizó el método de Sullivan ${ }^{21}$ y se llevó a cabo la modificación de la tabla de vida ajustada por la prevalencia de la discapacidad. A pesar de que este método tiene la desventaja de que supone que la mortalidad se comporta igual con discapacidad que sin ella, el presente estudio no tiene el objetivo de proporcionar un indicador que refleje diferentes condiciones de salud de la población en el tiempo y que permita discriminar estados absolutos como muerte o discapacidad.

En la estimación de EVISA, la medición de comorbilidad implicó el análisis de cada uno de los padecimientos y las secuelas discapacitantes de manera independiente; asimismo, se calculó la prevalencia de discapacidad para cada enfermedad, en la que los casos prevalentes fueron ajustados por la gravedad de las secuelas.

Si bien este estudio no recurrió a otras fuentes de información disponibles, consideramos que los datos obtenidos a partir de la información estadística institucional constituyen una fuente confiable en virtud de que dicha información está sujeta a un proceso continuo de mejora en cuanto al registro y la captura en línea de información, de forma que se garantice la calidad de los datos y se reduzcan al máximo las posibilidades de error. Por otra parte, la validez de la información institucional ha sido documentada ${ }^{22}$ y se ha dado seguimiento a diversos indicadores, lo que ha permitido encontrar una concordancia alta entre los registros, una consistencia interna aceptable y un promedio de casos discrepantes similar al que reportan otros países. Por tanto, puede decirse que aun cuando existan algunas limitaciones en el sistema de información institucional, éstas no alcanzan a afectar las conclusiones de este trabajo.

Esta investigación se llevó a cabo previa autorización del Comité de Investigación y de Ética de la Coordinación de Investigación en Salud del IMSS. Por otro lado, no se requirió de un consentimiento informado 
por tratarse de un análisis de información estadística de fuentes secundarias. Aun así, cabe mencionar que se tuvo especial cuidado en eliminar cualquier registro de afiliación a fin de evitar la identificación individual y atender por tanto las implicaciones éticas a las que se refiere la Declaración de Helsinki.

\section{Resultados}

Las estimaciones de la carga de la enfermedad para los derechohabientes se presentan en el cuadro I. En éste se comparan los resultados de los AVISA y la tasa correspondiente para los años 1995 y 2000 por causas seleccionadas. En ambos estudios, la mayor proporción de AVISA perdidos se debió a las enfermedades crónicas. En el año 2000, la tasa de AVISA resultó 1.5 veces mayor en comparación con 1995. Asimismo, en el año 2000 se observa un incremento de los años perdidos en los tres grupos de enfermedades. Destaca el hecho de que en el grupo 1 el incremento se explica por un aumento en los AVISA perdidos por SIDA y por trastornos hipertensivos del embarazo; este incremento coincide con un descenso en los AVISA por enfermedades diarreicas. En el grupo II, el incremento de los AVISA se debe a las afecciones de úlcera péptica, cáncer de mama, cáncer de próstata, diabetes mellitus y las enfermedades cerebro-vasculares; asimismo, llama la atención la disminución en la tasa por cáncer cervicouterino y el aumento en el peso de los trastornos depresivos. En el grupo III el aumento de AVISA se debe a las lesiones intencionales.

Como se observa en el cuadro II, las prioridades que se identifican al estimar los AVISA difieren de aquellas dictadas por la mortalidad. Ya en 1995 se identificaba el patrón de transición epidemiológica en la población derechohabiente, cuando la cardiopatía isquémica, la diabetes mellitus, la enfermedad cerebrovascular y la cirrosis hepática contrastaban con padecimientos tales como las afecciones perinatales y las infecciones respiratorias. Si bien estos padecimientos siguen siendo tan prioritarios en el año 2000 como cuando se considera sólo la mortalidad, en este caso se incorporan otras entidades nosológicas con una menor mortalidad, pero que al generar una discapacidad considerable constituyen una carga importante de enfermedad, tal como sucede con el SIDA, la depresión o las enfermedades perinatales.

La estimación de AVISA conjuga los panoramas contrastantes de la morbilidad y la mortalidad, e incorpora prioridades no consideradas por estos componentes, tales como los accidentes, la neumonía, el asma, la leucemia y la desnutrición en el caso de los niños, las lesiones no intencionales, la nefritis y la nefrosis, la leu- cemia en los adolescentes, los accidentes de trabajo en el hombre adulto, la depresión en hombres y mujeres adultos, el glaucoma y la enfermedad de Parkinson en el adulto mayor.

El mosaico nacional y la polarización epidemiológica observada en la población derechohabiente del IMSS se muestran en la figura 1. En ella se observa la contribución en APMP según el estrato epidemiológico para los años de 1995 y 2000. En general, los estados del sur del país presentan las tasas más altas del indicador de APMP y se ubican en el estrato de rezago extremo; en este caso se encuentran los estados de Chiapas, Oaxaca, Guerrero y Michoacán, entre otros. Por el contrario, los estados del norte del país se ubican en los estratos de la transición avanzada e intermedia, en los que las pérdidas por muerte prematura son 1.6 veces menores que las tasas de los estratos en rezago extremo y moderado.

El indicador de esperanza de vida al nacimiento en la población derechohabiente entre los años 1995 y 2000 se estimó en un rango entre 73.8 y 76.4 respectivamente, lo que implica un incremento promedio de 2.6 años en el periodo transcurrido entre los años de estudio. Al analizar la esperanza de vida al nacimiento por estratos epidemiológicos se constata que los estados en transición avanzada se ubicaron en un rango intercuartilar de 74.6-75.8 años en 1995, y de 76.7-77.9 años en 2000. Por el contrario, los estados en etapa de rezago epidemiológico quedaron ubicados en el cuartil más bajo, con un rango de 71.8-73.2 en 1995, y un rango de 73.1-75.8 años en 2000.

El indicador de EVISA para el año 1995 fue de 64.5 años, mientras que para el año 2000 fue de 65.8 años. En la figura 2 se muestra el indicador de Esperanza de vida (EV) y EVISA por estado en el año 2000. Considerando el fenómeno de transición, esta gráfica identifica la ubicación de las delegaciones de acuerdo con estos dos indicadores y permite observar diferencias importantes según la región del país y el estrato epidemiológico. La menor EV se estimó en 73.1 años para el estado de Chiapas (rezago extremo), mientras que la más alta fue de 79.8 años y correspondió al estado de Quintana Roo (transición avanzada). Estas diferencias son consistentes con el indicador EVISA que identifica el estado de Guerrero en etapa de rezago extremo con la menor esperanza de vida saludable (60.2 años) y el estado de Quintana Roo en etapa de transición avanzada con una EVISA de 68.5 años, y un promedio nacional de 65.5 años. Cabe señalar que el promedio del indicador de EV con discapacidad para la población IMSS en el año 2000 fue de 10.5 años, con un diferencial de 3.1 años más para las mujeres.

La figura 3 presenta dos mapas en los que se compara el avance de la transición epidemiológica en la po- 


\section{Cuadro I \\ Peso de la enfermedad en población derechohabiente del imSS por causas seleccionadas. MÉxıco, 1995 Y 2000}

\begin{tabular}{|c|c|c|c|c|}
\hline \multirow[b]{2}{*}{ Causas } & \multicolumn{2}{|c|}{1995} & \multicolumn{2}{|c|}{2000} \\
\hline & AVISA & Tasa* & AVISA & Tasa* \\
\hline Grupo I. Enfermedades infecciosas de la reproducción, perinatales y deficiencias nutricionales & 761008 & 2220 & I 284394 & 2867 \\
\hline Casos infectados con VIH sintomáticos & 58245 & 170 & 197038 & 440 \\
\hline Tuberculosis & 20561 & 60 & 16418 & 37 \\
\hline Infecciones respiratorias & 102972 & 300 & 176356 & 394 \\
\hline Enfermedades diarreicas & 41312 & 121 & 25921 & 58 \\
\hline Causas perinatales & 276747 & 807 & 536380 & 1197 \\
\hline Causas maternas & 52609 & 153 & 59476 & 273 \\
\hline Trastornos hipertensivos del embarazo & 13013 & 38 & 42691 & 196 \\
\hline Deficiencias nutricionales & 124490 & 363 & 58382 & 130 \\
\hline Grupo II. Enfermedades no transmisibles & 2401680 & 7006 & 5025530 & 11217 \\
\hline Neoplasias malignas & 354255 & 1033 & 743270 & 1659 \\
\hline Cáncer de mama & 34484 & 201 & 109465 & 503 \\
\hline Cáncer de traquea, bronquios, pulmón & 26916 & 79 & 64022 & 143 \\
\hline Cáncer cérvico-uterino & 42581 & 249 & 48120 & 221 \\
\hline Leucemia & 35505 & 104 & 60824 & 136 \\
\hline Cáncer de próstata & 13128 & 38 & 62030 & 138 \\
\hline Cáncer de colon y recto & 15887 & 46 & 43786 & 98 \\
\hline Cáncer de estómago & 21888 & 64 & 40575 & 91 \\
\hline Cáncer de esófago & 2735 & 8 & 5629 & 13 \\
\hline Linfoma y mieloma múltiple & 6650 & 19 & 24720 & 55 \\
\hline Melanoma y otros cánceres de piel & 5413 & 16 & 32840 & 73 \\
\hline Otras neoplasias & 12602 & 37 & 21893 & 49 \\
\hline Diabetes Mellitus & 272782 & 796 & 970593 & 2166 \\
\hline Enfermedades neuropsiquiátricas & 416230 & 1214 & 508284 & 1134 \\
\hline Trastornos depresivos & 100080 & 292 & 193389 & 432 \\
\hline Demencia y otros trastornos degenerativos y hereditarios del SNC & 65750 & 192 & 125155 & 279 \\
\hline Enfermedad de los órganos de los sentidos & 33683 & 98 & 140845 & 314 \\
\hline Glaucoma & 60338 & 176 & 110195 & 246 \\
\hline Enfermedades cardiovasculares & 390137 & 1138 & 999784 & 2231 \\
\hline Cardiopatía isquemia & 147934 & 432 & 350289 & 782 \\
\hline Enfermedad cerebrovascular & 90294 & 263 & 306503 & 684 \\
\hline Enfermedades respiratorias crónicas & 134375 & 392 & 197038 & 440 \\
\hline Enfermedad pulmonar obstructiva crónica & 56601 & 165 & 138656 & 309 \\
\hline Asma & 40723 & 119 & 58382 & 130 \\
\hline Enfermedades digestivas & 187055 & 546 & 437861 & 977 \\
\hline Cirrosis hepática & 97721 & 285 & 262717 & 586 \\
\hline Ulcera péptica & 7844 & 23 & 131358 & 293 \\
\hline Enfermedades genitourinarias & 130960 & 382 & 306503 & 684 \\
\hline Padecimientos músculo-esqueléticos & 139308 & 406 & 298232 & 666 \\
\hline Anomalías congénitas & 260292 & 759 & 328639 & 734 \\
\hline Enfermedades de la piel & 4037 & 12 & 20033 & 45 \\
\hline Grupo III. Lesiones & 7| 452 & 1667 & 966407 & 2157 \\
\hline Lesiones accidentales & 463459 & 1352 & 664090 & 1482 \\
\hline Lesiones intencionales & 107993 & 315 & 302317 & 675 \\
\hline Total $\left.\right|^{\ddagger}$ & $3734 I 40$ & 10892 & 7297691 & 16288 \\
\hline
\end{tabular}

* Tasa por 100000 derechohabientes

¥ Por ser causas seleccionadas, los totales de los grupos no corresponden a la suma de padecimientos

Fuente: Proyecto del peso de la enfermedad en el IMSS, 1995, y Proyecto Implantación de la Metodología de Esperanza de Vida Saludable en el IMSS, 2000. Análisis realizado en 2006 en el IMSS, México 
Cuadro II

Principales problemas de salud de la población derechohabiente del IMSS. México, 1995 y 2000

\begin{tabular}{|c|c|c|c|c|c|c|c|c|}
\hline \multirow[b]{2}{*}{ Prioridad } & \multicolumn{2}{|c|}{ Mortalidad* } & \multicolumn{2}{|c|}{$\begin{array}{c}\text { Años perdidos por muerte } \\
\text { prematura (APMP) }\end{array}$} & \multicolumn{2}{|c|}{$\begin{array}{c}\text { Años vividos con } \\
\text { discapacidad (AVD) }\end{array}$} & \multicolumn{2}{|c|}{$\begin{array}{c}\text { Años de vida saludables } \\
\text { perdidos (AVISA) }\end{array}$} \\
\hline & 1995 & 2000 & 1995 & 2000 & 1995 & 2000 & 1995 & 2000 \\
\hline I & $\begin{array}{l}\text { Cardiopatía } \\
\text { isquémica }\end{array}$ & $\begin{array}{l}\text { Diabetes } \\
\text { mellitus }\end{array}$ & $\begin{array}{l}\text { Afecciones } \\
\text { perinatales }\end{array}$ & $\begin{array}{l}\text { Diabetes } \\
\text { mellitus }\end{array}$ & $\begin{array}{l}\text { Diabetes } \\
\text { mellitus }\end{array}$ & $\begin{array}{l}\text { Diabetes } \\
\text { mellitus }\end{array}$ & $\begin{array}{l}\text { Afecciones } \\
\text { perinatales }\end{array}$ & $\begin{array}{l}\text { Diabetes } \\
\text { mellitus }\end{array}$ \\
\hline 2 & $\begin{array}{l}\text { Diabetes } \\
\text { mellitus }\end{array}$ & $\begin{array}{l}\text { Cardiopatía } \\
\text { isquémica }\end{array}$ & $\begin{array}{l}\text { Diabetes } \\
\text { mellitus }\end{array}$ & $\begin{array}{l}\text { Casos } \\
\text { infectados con } \\
\text { VIH sintomáticos }\end{array}$ & $\begin{array}{l}\text { Anomalías } \\
\text { congénitas }\end{array}$ & $\begin{array}{l}\text { Afecciones } \\
\text { perinatales }\end{array}$ & $\begin{array}{l}\text { Diabetes } \\
\text { mellitus }\end{array}$ & $\begin{array}{l}\text { Cardiopatía } \\
\text { isquémica }\end{array}$ \\
\hline 3 & $\begin{array}{l}\text { Enfermedad } \\
\text { cerebrovascular }\end{array}$ & $\begin{array}{l}\text { Enfermedad } \\
\text { cerebrovascular }\end{array}$ & $\begin{array}{l}\text { Accidentes de } \\
\text { vehículo de motor }\end{array}$ & $\begin{array}{l}\text { Cardiopatía } \\
\text { isquémica }\end{array}$ & $\begin{array}{l}\text { Trastornos } \\
\text { depresivos }\end{array}$ & $\begin{array}{l}\text { Cardiopatía } \\
\text { isquémica }\end{array}$ & $\begin{array}{l}\text { Cardiopatía } \\
\text { isquémica }\end{array}$ & $\begin{array}{l}\text { Afecciones } \\
\text { perinatales }\end{array}$ \\
\hline 4 & $\begin{array}{l}\text { Cirrosis } \\
\text { hepática }\end{array}$ & $\begin{array}{l}\text { Enfermedad } \\
\text { pulmonar } \\
\text { obstructiva crónica }\end{array}$ & $\begin{array}{l}\text { Cardiopatía } \\
\text { isquémica }\end{array}$ & $\begin{array}{l}\text { Enfermedad } \\
\text { cerebrovascular }\end{array}$ & $\begin{array}{l}\text { Accidentes de } \\
\text { vehículo de motor }\end{array}$ & $\begin{array}{l}\text { Enfermedad } \\
\text { cerebrovascular }\end{array}$ & $\begin{array}{l}\text { Accidentes } \\
\text { de vehículo } \\
\text { de motor }\end{array}$ & $\begin{array}{l}\text { Enfermedad } \\
\text { cerebrovascular }\end{array}$ \\
\hline 5 & $\begin{array}{l}\text { Afecciones } \\
\text { perinatales }\end{array}$ & $\begin{array}{l}\text { Cardiopatía } \\
\text { hipertensiva }\end{array}$ & $\begin{array}{l}\text { Homicidio } \\
\text { y violencia }\end{array}$ & $\begin{array}{l}\text { Cáncer } \\
\text { de mama }\end{array}$ & Osteoartritis & $\begin{array}{l}\text { Depresión } \\
\text { mayor unipolar }\end{array}$ & $\begin{array}{l}\text { Trastornos } \\
\text { depresivos }\end{array}$ & $\begin{array}{l}\text { Cirrosis } \\
\text { hepática }\end{array}$ \\
\hline 6 & $\begin{array}{l}\text { Enfermedad } \\
\text { pulmonar } \\
\text { obstructiva crónica }\end{array}$ & $\begin{array}{l}\text { Infecciones de } \\
\text { vías respiratorias } \\
\text { inferiores }\end{array}$ & $\begin{array}{l}\text { Nefritis } \\
\text { y nefrosis }\end{array}$ & $\begin{array}{l}\text { Cirrosis } \\
\text { hepática }\end{array}$ & $\begin{array}{l}\text { Dependencia } \\
\text { del alcohol }\end{array}$ & $\begin{array}{l}\text { Cirrosis } \\
\text { hepática }\end{array}$ & $\begin{array}{l}\text { Nefritis } \\
\text { y nefrosis }\end{array}$ & $\begin{array}{l}\text { Cardiopatía } \\
\text { hipertensiva }\end{array}$ \\
\hline 7 & $\begin{array}{l}\text { Infecciones de } \\
\text { vías respiratorias } \\
\text { inferiores }\end{array}$ & $\begin{array}{l}\text { Cirrosis } \\
\text { hepática }\end{array}$ & $\begin{array}{l}\text { Enfermedad } \\
\text { cerebrovascular }\end{array}$ & $\begin{array}{l}\text { Nefritis } \\
\text { y nefrosis }\end{array}$ & $\begin{array}{l}\text { Afecciones } \\
\text { perinatales }\end{array}$ & $\begin{array}{l}\text { Cardiopatía } \\
\text { hipertensiva }\end{array}$ & $\begin{array}{l}\text { Cirrosis } \\
\text { hepática }\end{array}$ & $\begin{array}{l}\text { Bajo peso } \\
\text { al nacer }\end{array}$ \\
\hline 8 & $\begin{array}{l}\text { Nefritis } \\
\text { y nefrosis }\end{array}$ & $\begin{array}{l}\text { Cáncer } \\
\text { cérvico-uterino }\end{array}$ & $\begin{array}{l}\text { Cirrosis } \\
\text { hepática }\end{array}$ & $\begin{array}{l}\text { Hipoxia } \\
\text { y trauma } \\
\text { al nacer }\end{array}$ & $\begin{array}{l}\text { Demencia senil } \\
\text { y otros trastornos } \\
\text { degenerativos SNC }\end{array}$ & $\begin{array}{l}\text { Bajo } \\
\text { peso al nacer }\end{array}$ & $\begin{array}{l}\text { Dependencia } \\
\text { del alcohol }\end{array}$ & $\begin{array}{l}\text { Casos infectados } \\
\text { con VIH } \\
\text { sintomáticos }\end{array}$ \\
\hline 9 & $\begin{array}{l}\text { Cardiopatía } \\
\text { hipertensiva }\end{array}$ & $\begin{array}{l}\text { Nefritis } \\
\text { y nefrosis }\end{array}$ & $\begin{array}{l}\text { Enfermedades } \\
\text { diarreicas }\end{array}$ & $\begin{array}{l}\text { Cardiopatía } \\
\text { hipertensiva }\end{array}$ & $\begin{array}{l}\text { Accidentes } \\
\text { de trabajo }\end{array}$ & $\begin{array}{l}\text { Accidentes } \\
\text { de trabajo }\end{array}$ & $\begin{array}{l}\text { Accidentes } \\
\text { de trabajo }\end{array}$ & $\begin{array}{l}\text { Nefritis } \\
\text { y nefrosis }\end{array}$ \\
\hline 10 & $\begin{array}{l}\text { Cáncer } \\
\text { pulmonar }\end{array}$ & $\begin{array}{l}\text { Cáncer } \\
\text { de mama }\end{array}$ & $\begin{array}{l}\text { Lesiones } \\
\text { auto infligidas } \\
\text { intencionalmente }\end{array}$ & $\begin{array}{l}\text { Lesiones } \\
\text { autoinfligidas } \\
\text { intencionalmente }\end{array}$ & $\begin{array}{l}\text { Nefritis } \\
\text { y nefrosis }\end{array}$ & $\begin{array}{l}\text { Demencia y otros } \\
\text { trastornos } \\
\text { degenerativos y } \\
\text { hereditarios del SNC }\end{array}$ & $\begin{array}{l}\text { Homicidio } \\
\text { y violencia }\end{array}$ & $\begin{array}{l}\text { Trastornos } \\
\text { depresivos }\end{array}$ \\
\hline
\end{tabular}

* Calculado por el indicador de muerte proporcional

Análisis realizado en 2006 en el IMSS, México

blación derechohabiente del IMSS en el periodo de estudio. Así, por ejemplo, el estado de Michoacán pasó de rezago extremo en 1995 a rezago infantil en 2000; Baja California, Sinaloa y Aguascalientes pasaron de rezago infantil en 1995 a transición intermedia en el año 2000; los estados de Nayarit, Quintana Roo y Coahuila, que en 1995 se encontraban en transición intermedia, pasaron a una etapa de transición avanzada en 2000; por último, algunos estados tales como el Estado de México, Chiapas, Guerrero, Hidalgo, Oaxaca, Yucatán y Zacatecas se mantuvieron en 2000 en la misma posición respecto de 1995.

\section{Discusión}

El uso en este estudio de indicadores compuestos tales como AVISA permitió identificar el cambio en el perfil de la salud de los derechohabientes y la forma en que sus condiciones de salud se han modificado en el curso del tiempo. Mostró asimismo que el solo uso de la morbilidad y la mortalidad no permite identificar las prioridades de atención de la salud.

La morbilidad en el Instituto, documentada por la demanda de consulta y los egresos hospitalarios, expresa la demanda percibida de atención, así como la expresión clínica o la gravedad de algunas entidades nosológicas que ameritan hospitalización. Otras enfermedades expresan su ocurrencia en la mortalidad observada. No obstante, este estudio de la carga de la enfermedad permitió identificar padecimientos que, sin ocupar los primeros lugares como causa de consulta, hospitalización o mortalidad, representan una carga importante en la salud de la población protegida por el Instituto Mexicano del Seguro Social.

Una de las prioridades observadas que más llama la atención es la infección por VIH que, si bien se ubica dentro de las 10 prioridades por AVISA, se encuentra ausente dentro de las principales causas de morbilidad o mortalidad. La importante contribución de años perdidos por muerte prematura secundarios al SIDA refleja lo 


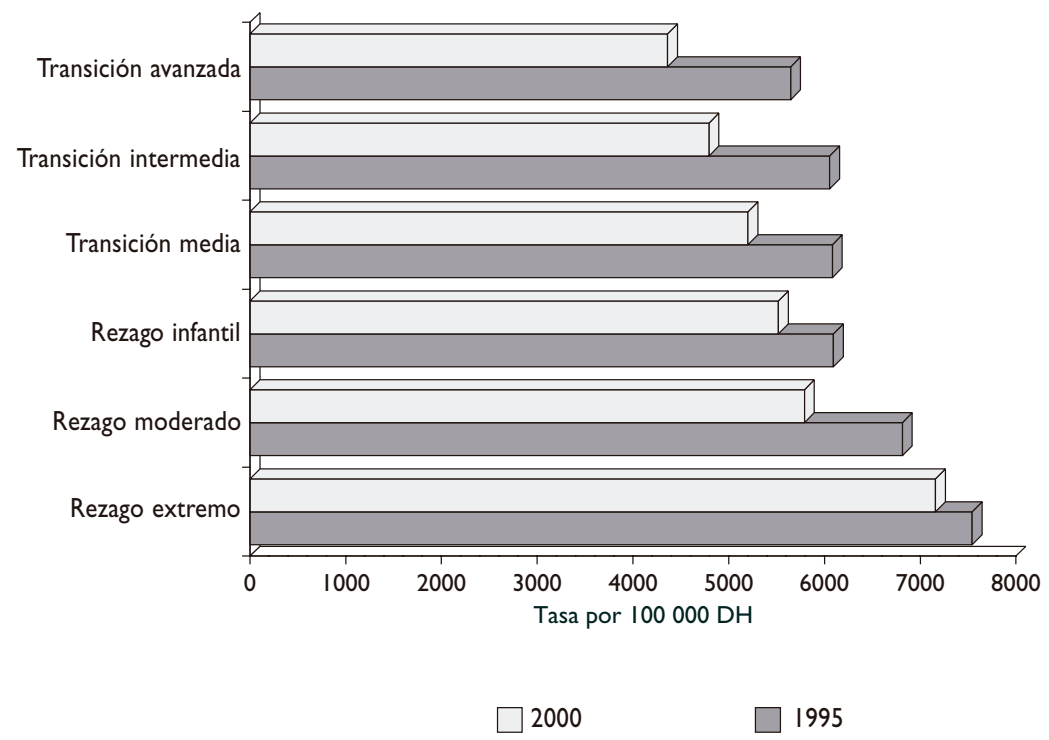

Ubicación según transición de la mortalidad por entidad federativa

1995: Transición avanzada: Sonora, Chihuahua, DF, Tamaulipas, Baja California Sur, Nuevo León Transición intermedia: Quintana Roo, Coahuila, Nayarit,Veracruz Sur Transición media:Tabasco, Colima, Estado de México, Jalisco Rezago infantil: Sinaloa, Yucatán, Campeche, Morelos, Tlaxcala, Baja California, Aguascalientes Rezago moderado: Puebla, Veracruz Norte, Zacatecas, Durango, Hidalgo, San Luis Potosí, Querétaro Rezago extremo: Guanajuato, Michoacán, Oaxaca, Guerrero, Chiapas.

2000: Transición avanzada: Sonora, Chihuahua, DF, Tamaulipas, Baja California Sur, Nuevo León, Coahuila, Quintana Roo, Nayarit, Jalisco Transición intermedia:Veracruz, Tabasco, Colima, Aguascalientes, Sinaloa, Baja California, Querétaro Transición media: Estado de México, Morelos, Campeche, Tlaxcala, Durango, San Luis Potosí Rezago infantil:Yucatán, Michoacán Rezago moderado: Guanajuato, Zacatecas, Hidalgo

Rezago extremo: Chiapas, Puebla, Oaxaca, Guerrero

Análisis realizado en 2006 en el IMSS, México

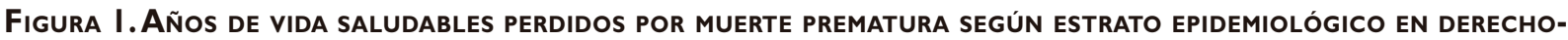
habientes del IMSS. MéXICO, I 995 y 2000

tardío del tratamiento e, incluso, del diagnóstico, condición que se agrava si se considera la elevada letalidad del padecimiento. Otra prioridad de salud identificada con la estimación de AVISA y que no se ubica dentro de las primeras causas de morbilidad y mortalidad fue la depresión. Con una contribución del $2.65 \%$ de los AVISA perdidos, la depresión ha sido un padecimiento constante para el que se han realizado diferentes estimaciones de carga de la enfermedad, con participaciones que oscilan entre $3.7 \%$ en Australia, ${ }^{23} 4.4 \%$ en Holanda ${ }^{7}$ y $6.9 \%$ en Ginebra. ${ }^{24} \mathrm{Si}$ bien se ha propuesto que la carga condicionada por la depresión ha sido sobreestimada, no existe evidencia de que la discapacidad asociada con la depresión haya sido considerada de igual forma. ${ }^{25}$
Sin duda alguna, la diabetes mellitus tipo 2 es la enfermedad que representa la mayor carga en la salud de los mexicanos amparados por el Instituto Mexicano del Seguro Social. Es la primera causa de mortalidad, la primera causa de años perdidos por muerte prematura y la primera de años vividos con discapacidad. Es la principal causa de AVISA y contribuye con 13\% del total de AVISA perdidos por la población derechohabiente. Entre 2003 y 2005, el costo de atención de la diabetes en México sobrepasó los 300 millones de dólares, de los que 60\% correspondió al gasto del IMSS en esta enfermedad. ${ }^{26}$ La diabetes representa una carga importante para países en desarrollo como México, carga que seguramente se incrementará cuando el nú- 


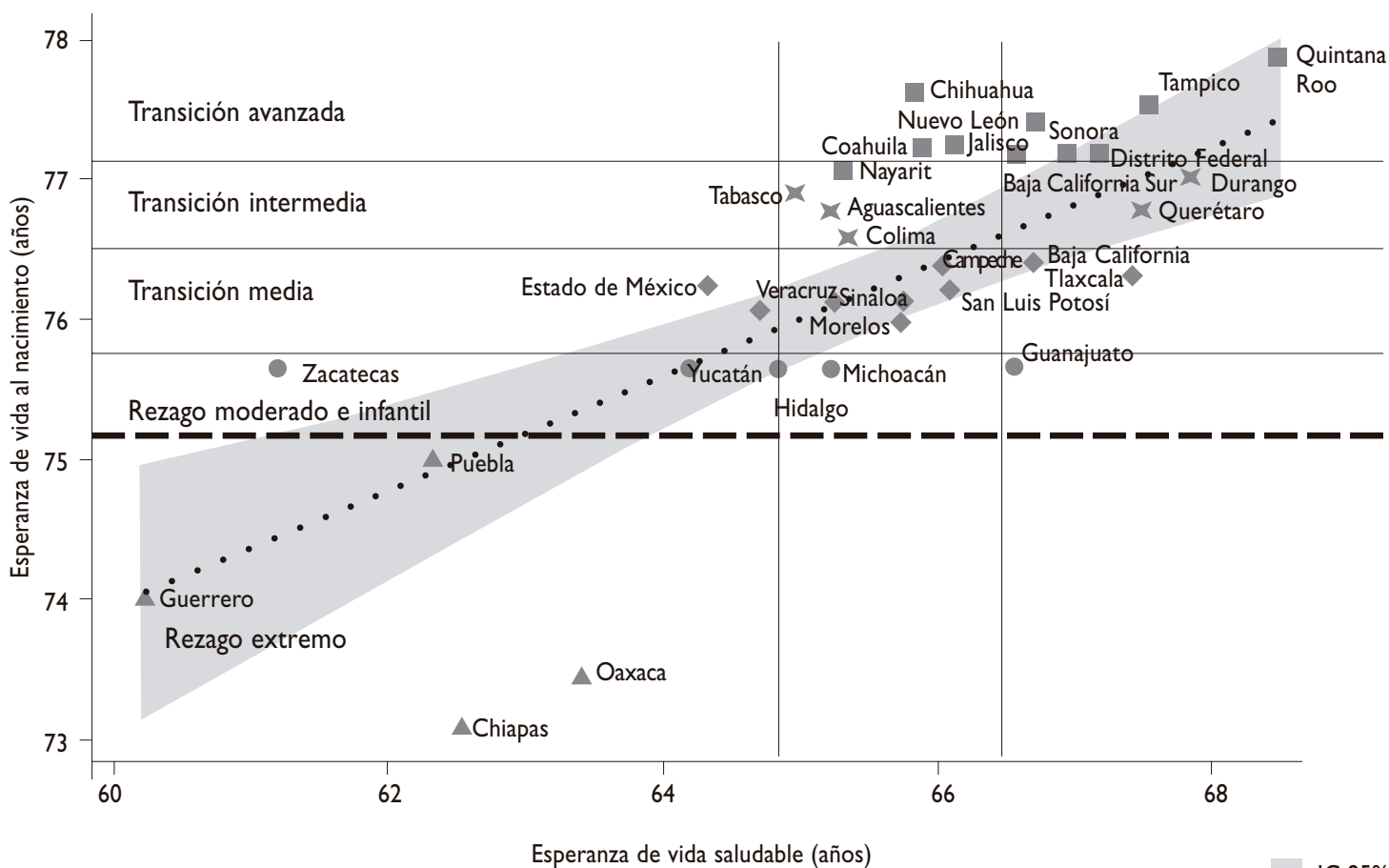

Análisis realizado en 2006 en el IMSS, México

IC $95 \%$

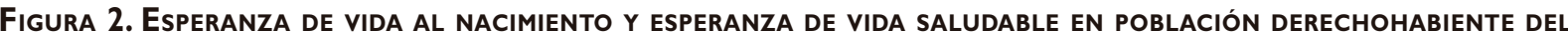
IMSS SEgún ETAPAS de transición. MÉxico, 2000

mero de diabéticos se duplique al término de los próximos 25 años. ${ }^{27}$

Junto con la diabetes mellitus, la cardiopatía isquémica representa también una importante carga de enfermedad. Destaca el hecho de que en hombres y mujeres en edad productiva (20 a 59 años) representa la segunda causa de pérdida de AVISA, aun cuando no ocupe ese lugar ni en morbilidad ni en mortalidad. La epidemia de la enfermedad cardiovascular se extiende de forma importante en los países en vías de desarrollo. La exposición al tabaco se ha incrementado en las naciones en desarrollo como México, ${ }^{28}$ y esta exposición es mayor en la población de menores recursos. ${ }^{29}$ En el estudio se identificó la enfermedad cerebrovascular como la principal causa de pérdida de AVISA en el adulto mayor, si bien ocupa también un lugar importante en la población en edad productiva, lo que traslada la ocurrencia de los eventos cardiovasculares y cerebrovasculares a edades más tempranas.

Si bien la contribución porcentual de las enfermedades infecciosas, de la reproducción, perinatales y de las deficiencias nutricionales descendió de 19.2\% en 1995 a 17.6\% en el año 2000, lo cierto es que éstas siguen siendo una causa importante de pérdida de AVISA, sobre todo ante el incremento observado en los AVISA perdidos por SIDA. En este caso, la proporción observada es menor que la notificada por otros países en desarrollo que han estimado la carga de la enfermedad por AVISA, ${ }^{30}$ pero se encuentra aún lejos de $6.6 \%$ observado en países desarrollados del continente Americano como los Estados Unidos, Cuba y Canadá. ${ }^{31}$

Las lesiones no intencionales son una causa importante de pérdida de AVISA en población joven, fenómeno que se ha observado en otras poblaciones. ${ }^{21}$ De hecho, la contribución porcentual de las lesiones en los países desarrollados ha superado ya la observada por las enfermedades infecciosas, de la reproducción, perinatales y por deficiencias nutricionales. ${ }^{7,20,28,32}$ Por su parte, la enfermedad cerebrovascular resultó ser en este estudio una de las principales causas de pérdida de AVISA en el adulto mayor; al respecto, se ha documentado que esta enfermedad es la principal causa de discapacidad en los países en desarrollo. ${ }^{33}$ 


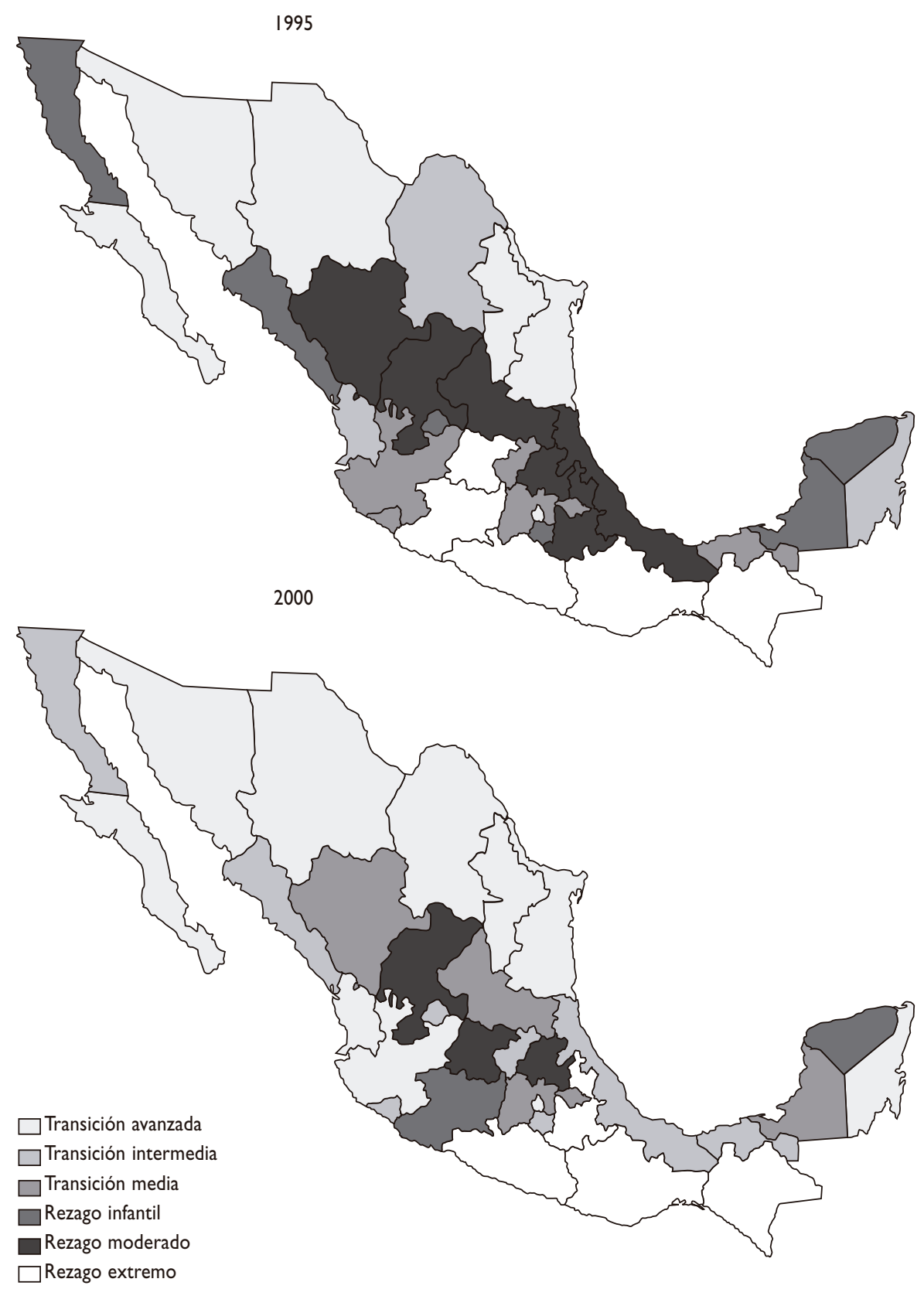

Análisis realizado en 2006 en el IMSS, México

Figura 3. Cambios en las condiciones de salud de los derechohabientes del imSS según la transición de la MORTALIDAD POR ENTIDAD Federativa. México 1995- 2000

La importancia de trastornos de la visión en este grupo de edad, tales como las cataratas o el glaucoma, resaltan un problema de salud que requiere mayor vigilancia y control.

La carga de la enfermedad difiere no sólo entre poblaciones, sino al interior de un mismo país. La po- larización epidemiológica es un componente de la transición epidemiológica que experimentan países como México. Las diferencias observadas entre la contribución porcentual de los años perdidos por muerte prematura y aquellos vividos con discapacidad entre los diferentes estados del país ponen de manifiesto 
las diferentes condiciones socioeconómicas y de marginalidad. Estas diferencias permiten identificar dos patrones en las condiciones de salud-enfermedad, el de la pobreza y el rezago y el del desarrollo, que son una expresión clara de la existencia de desigualdades e inequidades aún no resueltas en el contexto nacional. ${ }^{34}$ La carga de la enfermedad se encuentra estrechamente relacionada con las diferencias socioeconómicas ${ }^{35} \mathrm{y}$ el ingreso de las poblaciones. ${ }^{36}$ Son varias las condiciones que contribuyen a estas disparidades, pero son unas pocas las entidades nosológicas que tienen la mayor incidencia en la carga de la enfermedad, tales como las que se relacionan con el tabaquismo (cardiopatía isquémica, cáncer de pulmón), la hipertensión, la diabetes mellitus, las lesiones y la infección por $\mathrm{VIH} .{ }^{37} \mathrm{La}$ esperanza de vida se ve indudablemente afectada por la presencia de estas condiciones, al igual que la esperanza de vida ajustada por discapacidad.

Cabe mencionar que los resultados del indicador EVISA obtenidos en el presente trabajo identifican ciertos estados en estratos que podrían no corresponder al patrón epidemiológico esperado. Al respecto, Rodríguez y col..$^{38}$ llevan a cabo una comparación entre esperanza de vida al nacimiento y esperanza de vida libre de discapacidad y mencionan que éstas se ven indudablemente afectadas por diversas condiciones patológicas que inciden en la mortalidad, la morbilidad y la discapacidad, lo que influye en un diferencial identificado como la brecha en salud, que idealmente se tendría que reducir y que corresponde a la esperanza de vida con discapacidad. Con ambos indicadores se tratar de discriminar las condiciones de salud e identificar diferencias entre los estados con mayor desarrollo y aquéllos con un desarrollo intermedio que, si bien han mejorado su condición de vida saludable, aún conservan un componente asociado con discapacidad que influye en el avance de su salud.

Las políticas de salud de una nación deben estar dirigidas a intervenir en aquellas condiciones de salud que representan una mayor carga para su población. La identificación de estas prioridades en la población amparada por el Instituto Mexicano del Seguro Social debe servir para orientar y/o fortalecer el desarrollo de políticas de salud específicas, por grupo de edad y por región en el país. Asimismo, la adopción de políticas basadas en evidencias permitirá evaluar el impacto de los programas en operación con la finalidad de mejorar la gestión y asignación de recursos, promover el financiamiento para la investigación en áreas identificadas y reducir así las brechas existentes entre los diferentes grupos de población.

\section{Referencias}

I. Lozano R, Murray CJL, Frenk J, Bobadilla JL. Burden of disease assessment and health system reform: results of a study in Mexico. J Int Dev 1995; 7:555-563.

2. Frenk J, Lozano R, González-Block MA, Ruelas E, Bobadilla JL. Economía y Salud: Propuestas para el avance del sistema de salud en México. México:Fundación Mexicana para la Salud, 1994.

3. Seguro Popular. Disponible en :: www.salud.gob.mx/unidades/dgpfs 4. The World Bank. World Development Report 1993. Investing in health. Washington, DC: Oxford University Press, 1993.

5. World Health Organization. Investing in health research and development. Report of the Ad Hoc Committee on health research relating to future intervention options. Ginebra:WHO, 1996. 6. Murray CJL, Lopez AD. Global health statistics: a compendium of incidence, prevalence and mortality estimates for over 200 conditions. Cambridge: Harvard University Press, 1996.

7. Melse JM, Essink-Bot ML, Kramers PGN, Hoeymans N. On behalf of the Dutch Burden of Disease Group.A national burden of disease calculation: Dutch disability-adjusted life-years. Am J Public Health 2000;90: I 24I-I 247.

8. Murray CL, Lopez AD. Regional patterns of disability-free life expectancy and disability-adjusted life expectancy: Global Burden of Disease Study. Lancet 1997;349: I 347-I 352.

9. Mathers CD, Sadana R, Salomon JA, Murray CJ, Lopez AD. Healthy life expectancy in 191 countries, 1999. Lancet 2001; 357: 1685-1691. 10. Organización Panamericana de la Salud. Clasificación estadística internacional de enfermedades y problemas relacionados con la salud. 9na revisión. Washington: OPS, 1975.

II. Organización Panamericana de la Salud. Clasificación estadística internacional de enfermedades y problemas relacionados con la salud. IOma revisión. Washington: OPS, 1995.

12. Murray CJL. Cuantificación de la carga de enfermedad: la base técnica del cálculo de los años de vida ajustados en función de la discapacidad. Bol Oficina Sanit Panam 1995; I 18(3):221-24I.

13. Murray CJ. Review of DALYs. En: Murray CL, Michaud C, Mahapatra P, Acharya A, Shibuya K, Lozano R, et al. Designing and implementing a National Burden of Disease Study. Burden of Disease. Boston: Harvard Center for Population and Development Studies, 1995:37-75.

14. Murray CJ.Validity of cause of death attribution and practical methods for cause adjustments. En: Michaud C, Mahapatra P, Acharya A, Shibuya K, Lozano R, et al. Designing and implementing a National Burden of Disease Study. Burden of Disease. Boston: Harvard Center for Population and Development Studies, 1995:91-1 18.

15. Brass W. Methods of Demographic Estimation from Incomplete Data. Laboratories for Population Statistics. USA. Chapel Hill: University of North Carolina, 1975.

16. Coale AJ, Demeny P, Vaughan B. Models of mortality and age composition. En: Coale AJ, ed. Regional Model Life Tables and Stable Population. 2nd edition. New York: Academic Press, 1983:3-7.

17. Coale A, Trussell T. Model Fertility Schedules:Variations in the Age Structure of Childbearing in Human Population. Population Index 1974;40(2):185-258.

18. United Nations. MortPak-Lite:The United Nations Software Package for Mortality Measurement. New York: Population Studies 104, 1988.

19. Chiang CH. Life Tables and Mortality Analysis. Ginebra:World Health Organization, 1978.

20. Lozano R. El peso de la enfermedad en México, avances y desafíos. En: Frenk J. Observatorio de la salud. Necesidades, servicios y políticas. México: Fundación Mexicana para la Salud, 1994: 23-61. 
21. Mathers CD, Sadana R, Salomon JA, Murray CJL, Lopez AD. Estimates of DALE for 191 countries: methods and results. Global Programme on Evidence for Health Policy Working Paper No. 16. Ginebra:World Health Organization, 2000.

22. Coria I, Mata J,Acevedo F, Recio M, Zurita B, Esquivel G. La calidad de la información del expediente clínico como herramienta para la integración de los grupos relacionados de diagnóstico (GRD). En: Martínez-Salgado H, Villasís MA, Torres J, Gómez-Delgado A, eds. Las múltiples facetas de la investigación en Salud 4. México DF: Instituto Mexicano del Seguro Social, 2000.

23. Mathers CD, Vos ET, Stevenson CE, Begg SJ. The burden of disease and injury in Australia. Bull World Health Organ 200I; 79: 1076-1084. 24. Schopper D, Pereira J, Torres A, Cuende N,Alonso M, Baylin A, et al. Estimating the burden of disease in one Swiss canton: what do disability adjusted life years (DALY) tell us? Int J Epidemiol 2000; 29:87I-877. 25. Kruijshaar ME, Hoeymans N, Spijker J, Stouthard MEA, Essink-Bot ML. Has the burden of depression been overestimated? Bull World Health Organ 2005; 83:443-448.

26. Arredondo A, Zúñiga A. Economic consequences of epidemiological changes in diabetes in middle-income countries. The Mexican case. Diabetes Care 2004; 27:104-109.

27. Roglic G, Unwin N, Bennett PH, Mathers C, Tuomilehto J, Nag S, et al. The burden of mortality attributable to diabetes. Diabetes Care 2005; 28:2/30-2135.

28. Fernández-Gárate IH, Escobedo-de la Peña J, Hernández-Tamayo D, Tudón-Garcés H, Ramírez-Galindo JD, Benítez-Martínez MG, et al. Consumo de tabaco en la población derechohabiente del Instituto Mexicano del Seguro Social (Tobacco consumption among the active insured population of the Mexican Social Security Institute). Salud Publica Mex 1997; 39:125-132.
29. Reddy K S. Cardiovascular disease in non-western countries. N Engl J Med 2004;350:2438-2440.

30. Hyder AA, Morrow RH. Applying burden of disease methods in developing countries: a case study from Pakistan. Am J Public Health 2000;90:1235-1240.

31.World Health Organization. The World Health Report 2004: changing history. Ginebra:World Health Organization, 2004. 32. McKenna MT, Michaud CM, Murray CJ, Marks JS. Assessing the burden of disease in the United States using disability-adjusted life years. Am J Prevent Med 2005;28:415-423.

33. Truelsen T, Begs S, Mathers C. The global burden of cerebrovascular disease. Global Burden disease 2000. Ginebra:World Health Organization, 2000.

34. Zurita B, Lozano R, Ramírez T, Torres JL. Desigualdad e inequidad en salud. En: Knaul F, Nigenda G, eds. Caleidoscopio de la Salud. De la investigación a las políticas y de las políticas a la acción. México: Fundación Mexicana para la Salud, 2003.

35. Ljung R, Peterson S, Hallqvist J, Heimerson I, Diderichsen F. Socioeconomic differences in the burden of disease in Sweden. Bull World Health Organ 2005;83:92-99.

36. Muennig P, Franks P, Jia H, Lubetkin E, Gold MR. The incomeassociated burden of disease in the United States. Soc Sci Med 2005;61:2018-2026.

37. Wong MD, Shapiro MF, Boscardin WJ, Ettner SL. Contribution of major diseases to disparities in mortality. N EngI J Med 2002;347:15851592.

38. Rodríguez AG, Escobedo -de la Peña J, Zurita GB, Ramírez MT. Esperanza de vida saludable en la población mexicana con seguridad social. Perinatal Reprod Hum 2006: 20(I-3): 4-I8. 